\title{
Relation between the grafting density of liquid crystal macromolecule and the symmetry of self-assembled bulk phase: coarse-grained molecular dynamics study
}

\author{
J.M. Ilnytskyi \\ Institute for Condensed Matter Physics of the National Academy of Sciences of Ukraine,
} 1 Svientsitskii St., 79011 Lviv, Ukraine

Received August 20, 2013

\begin{abstract}
I consider a generic coarse-grained model suitable for the study of bulk self-assembly of liquid crystal (LC) macromolecules. The cases include LC dendrimers, gold nanoparticles modified by polymer chains with terminating LC groups and others. The study is focused on the relation between a number of grafted chains, $N_{\mathrm{ch}}$, and the symmetry of the self-assembled bulk phases. Simple space-filling arguments are used first to estimate stability intervals for a rod-like, disc-like and spherulitic conformations in terms of $N_{\mathrm{ch}}$. These are followed by coarse-grained molecular dynamics simulations for both spontaneous and aided self-assembly of LC macromolecules into bulk phases. In spontaneous self-assembly runs, essential coexistence of rod-like and disc-like conformations is observed (via analysis of the histograms for the molecular asphericity) in a broad interval of $N_{\mathrm{ch}}$, which prevents the formation of defect-free structures. The use of uniaxial and planar aiding fields is found to improve self-assembly into monodomain phases by promoting conformations of respective symmetry. Strong shape-phase relation, observed experimentally, is also indicated by the simulations by the coincidence of the stability intervals for the respective conformations with those for the bulk phases.
\end{abstract}

Key words: macromolecules, liquid crystals, self-assembling, molecular dynamics structure effects on the order

PACS: $02.70 . N s, 61.30 . V x, 61.30 . C z, 61.30 . G d$

\section{Introduction}

When different (in size, shape or interaction potential) molecular fragments are combined into a single molecule, then one obtains the so-called polyphilic material [1, 2] (the simplest example being well-known amphiphiles). If such a molecule is big enough, then it represents a supermolecular object, although the supramolecular effects are also possible via self-assembly of polyphilic macromolecules into bulk ordered phases [3-6]. The self-assembly is predominantly driven by a microphase separation, which, in turn, depends on both the details of molecular architecture and on the level of chemical compatibility between the constituent parts of a molecule.

LC polyphilic macromolecules incorporate mesogenic groups in addition to branched polymer chains, nanoparticles, etc. Variation of a molecular architecture gives rise to main- or side-chain LC polymers, LC dendrimers and elastomers, LC gold metamaterials [7-10]. The microphase separation in such systems originates from poor miscibility of aromatic and aliphatic fragments, and/or size differences between them (e.g., larger nanoparticle and smaller polymer bead or mesogen) as well as on the other details of interparticle interactions.

Despite a broad variety of possible molecular architectures, certain cases bear prominent similarities. Good example is provided by the existence of many common features in self-assembly of LC dendrimers and LC gold metamaterials [8, 10-12], and I will concentrate on these particular cases in this study. Both 
systems exhibit a similar set of lamellar, columnar and various cubic phases [8, 10-17]. Strictly speaking, the interior of these types of macromolecules is rather different: flexible hyperbranched polymer scaffold vs solid nanoparticle, respectively. This implies a different type of grafting in each case: more of annealed type for LC dendrimer (the level of rearrangement freedom for grafted beads depends on the dendrimer generation [13, 14]), and more of quenched type for gold metaparticle. Nevertheless, the general aspects of a self-assembly turned out to be more dependent on the space filling capabilities of grafted chains and on the strength of the mesogen-mesogen interaction [8, 11, 12] than on the details of internal structure of the macromolecule.

The similarities between the LC dendrimers and LC gold metaparticles open up a possibility to describe their self-assembly by some generic coarse-grained model, in which less relevant internal degrees of freedom of a central core are neglected and only rearrangement of the attached polymer chains with terminating mesogens is taken into account. The grounds for such coarse-graining (besides general arguments given above) are to be found in some previous simulation studies [18-20], where, in particular, it was found that the central core of the generation three carbosilane dendrimer is on average spherically symmetric in all (isotropic, nematic and smectic A) phases of LC solvent [18]. The models that exploit this fact have already been considered, namely in the form of a sphere with attached chains, each containing a mesogen [21, 22], as well as a sphere decorated by Gay-Berne particles directly on its surface [23]. The number of bulk phases have been found in these simulation works. The models, however, permit tuning in a number of ways (the number and length of grafted chains, the precise way of grafting, the way terminal mesogens are attached, etc.) and the effects of all these changes still await to be analysed in detail by computer simulations.

The experimental studies reveal the existence of strong dependence between the density of mesogens on the macromolecule surface, molecular conformation in bulk phase and the symmetry of the latter [8, 10-17]. As remarked in reference [8], the grafting density "can effectively change the overall gross shape of the structure of the supermolecule from being rod-like, to disc-like, to spherulitic. Thus, the structure of the systems at a molecular level can be considered as being deformable, where each type of molecular shape will support different types of self-organized mesophase structure. Thus, for supermolecular materials, rod-like systems will support the formation of calamitic mesophases (including various possibilities of smectic polymorphism), disc-like systems tend to support columnar mesophases, and spherulitic systems form cubic phases”. The current study addresses this effect by means of computer simulation.

The work is a continuation of the study performed in reference [22], where the coarse-grained generic model for the LC metaparticle was introduced and studied on a subject of a bulk self-assembly. The model contained a central sphere and 32 free-sliding chains each terminated by a mesogen and the simulations were performed by means of a coarse-grained molecular dynamics (CGMD). It has been found that the melt self-assemble into either smectic lamellar or hexagonal columnar morphology when aided briefly by an uniaxial or planar external field, respectively. The molecular shape is predominantly rod-like in the smectic phase and disc-like in the columnar one. This shape bistability was analysed by means of average metric properties (gyration tensor, average asphericity, etc.). On the contrary, the unaided (spontaneous) self-assembly by means of either slow compression or cooling the melt down was found to always result in the polydomain phase. Here, these findings are extended in two directions. Firstly, the model is generalized to the case of an arbitrary number of grafted chains $N_{\mathrm{ch}}$, which allows one to study the intervals of stability for each bulk phase on $N_{\text {ch }}$ by means of CGMD simulations. Secondly, both aided and spontaneous self-assembly is analysed in detail by splitting the melt into subsystems of rod-like and disc-like molecules and monitoring the histograms of their asphericities. The phase boundaries obtained by means of CGMD are also compared with the results of purely geometric analysis for athermal space-filled rod, disc and sphere.

The following section contains a description of the model and a space-filling analysis. Section 3 contains the results for the CGMD simulations of the bulk phases by means of spontaneous and aided selfassembly, as well as a detailed analysis of molecular conformations. Conclusions are provided in section 4 


\section{Modelling and computational details}

The coarse-grained model for LC dendrimer or LC gold metaparticle (hereafter referred to as "generic model”) is depicted schematically in figure 1 Large central sphere represents a coarse-grained core of a macromolecule with its internal degrees of freedom being neglected. Four smaller spheres (each being a fragment of a polymer chain of a few hydrocarbons) form a spacer. The latter is terminated by a spherocylinder representing a coarse-grained mesogenic (LC) group.

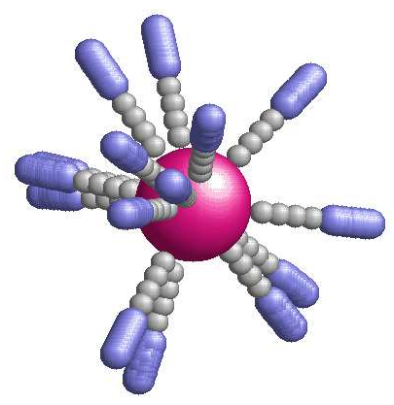

Figure 1. (Color online) Generic coarse-grained model of liquid crystal colloid consisting of large central sphere and $N_{\mathrm{ch}}$ freely-grafted chains each terminated by a mesogen.

This model, introduced in reference [22] and studied there for the case of $N_{\mathrm{ch}}=32$ attached chains only, is generalised here for the case of arbitrary $N_{\mathrm{ch}}$. The first bead of each chain can be attached to the surface of a central sphere in a number of ways, in particular: (i) quenched-like grafting to a particular point on a surface; (ii) semi-quenched-like grafting with the employment of an angular elastic spring with respect to a particular point; (iii) annealead-like grafting, when the end bead is capable of sliding freely on the surface. In all cases, radial elastic spring can be used to ensure that the first bead is always located on the surface of a large sphere. It is evident that option (i) would be the best suited to model the LC gold metaparticle, whereas option (ii) would represent the LC dendrimer (e.g., such option was applied in [21]). Option (iii) can be seen as some limit case representing the infinite generation LC dendrimer or the metamolecule with an additional symmetry of chains interexchange. The latter is not unreasonable for the equilibration speed-up and is, in fact, on par with high interpenetrability of soft beads in CGMD modelling employed here. The option (iii) with annealed grafting is used in this study.

The effective dimensions of soft beads are based on the coarse-graining of the atomistic model for the generation $3 \mathrm{LC}$ dendrimer [21]. These are: $\sigma_{1}=21.37 \AA$ for a large sphere, $\sigma_{2}=6.23 \AA$ for the first bead of a spacer, $\sigma_{3}=4.59 \AA$ for all the rest beads of the spacer and $D=3.74 \AA, L / D=3$ for the mesogen breadth and elongation, respectively. These dimensions are also used for the visualisation purpose. The interaction potential between any two spheres has a quadratic form:

$$
V_{i j}^{\mathrm{sp}-\mathrm{sp}}= \begin{cases}U_{\max }^{\mathrm{sp}-\mathrm{sp}}\left(1-r_{i j}^{*}\right)^{2}, & r_{i j}^{*}<1, \\ 0, & r_{i j}^{*} \geqslant 1,\end{cases}
$$

where $r_{i j}^{*}=r_{i j} / \sigma_{i j}$ is the scaled distance between the centers of $i$-th and $j$-th sphere, and usual mixing rules $\sigma_{i j}=\left(\sigma_{i}+\sigma_{j}\right) / 2$ are employed for the spheres with different diameters $\sigma_{i}$ and $\sigma_{j}$. The value of $U_{\max }^{\mathrm{sp}-\mathrm{sp}}=70 \cdot 10^{-20} \mathrm{~J}$ is the same for all combinations of interacting spheres. The same potential form is used for interaction between the sphere and the spherocylinder:

$$
V_{i j}^{\mathrm{sp}-\mathrm{sc}}= \begin{cases}U_{\max }^{\mathrm{sp}-\mathrm{sc}}\left(1-d_{i j}^{*}\right)^{2}, & d_{i j}^{*}<1, \\ 0, & d_{i j}^{*} \geqslant 1,\end{cases}
$$

where $d_{i j}^{*}=d_{i j} / \sigma_{i j}$ is a dimensionless closest distance between the center of the $i$-th sphere and the core of the $j$-th spherocylinder, with the scaling factor $\sigma_{i j}=\left(\sigma_{i}+D\right) / 2$. Parameter $U_{\max }^{\mathrm{sp}-\mathrm{sc}}$ is equal to $U_{\max }^{\mathrm{sp}-\mathrm{sp}}$ (see above). 
Spherocylinder-spherocylinder pairwise interaction has the form introduced by Lintuvuori and Wilson [24]:

$$
V_{i j}^{\mathrm{sc}-\mathrm{sc}}= \begin{cases}U_{\max }^{\mathrm{sc}-\mathrm{sc}}\left(1-d_{i j}^{*}\right)^{2}+\epsilon^{*}, & d_{i j}^{*}<1, \\ U_{\max }^{\mathrm{sc}-\mathrm{sc}}\left(1-d_{i j}^{*}\right)^{2}-U_{\mathrm{attr}}^{*}\left(\hat{r}_{i j}, \hat{e}_{i}, \hat{e}_{j}\right)\left(1-d_{i j}^{*}\right)^{4}+\epsilon^{*}, & 1 \leqslant d_{i j}^{*}<d_{\mathrm{c}}^{*}, \\ 0, & d_{i j}^{*}>d_{\mathrm{c}}^{*},\end{cases}
$$

where $d_{i j}^{*}=d_{i j} / D$ is the dimensionless nearest distance between the cores of spherocylinders [25], $d_{\mathrm{c}}^{*}$ is the effective cutoff distance for the attractive interaction that depends on the attractive part of the potential

$$
U_{\mathrm{attr}}^{*}\left(\hat{r}_{i j}, \hat{e}_{i}, \hat{e}_{j}\right)=U_{\mathrm{attr}}^{*}-\left[5 \epsilon_{1} P_{2}\left(\hat{e}_{i} \cdot \hat{e}_{j}\right)+5 \epsilon_{2}\left(P_{2}\left(\hat{r}_{i j} \cdot \hat{e}_{i}\right)+P_{2}\left(\hat{r}_{i j} \cdot \hat{e}_{j}\right)\right]\right.
$$

The latter depends on the orientations $\hat{e}_{i}, \hat{e}_{j}$ of the long axes of spherocylinders and the unit vector $\hat{r}_{i j}$ that connect their centers, as discussed in more detail elsewhere [24]. $P_{2}(x)=1 / 2\left(3 x^{2}-1\right)$ is the second Legendre polynomial, the energy parameters are as follows: $U_{\max }^{\mathrm{sc}-\mathrm{sc}}=70 \cdot 10^{-20} \mathrm{~J}, U_{\mathrm{attr}}^{*}=1500 \cdot 10^{-20} \mathrm{~J}$, $\epsilon_{1}=120 \cdot 10^{-20} \mathrm{~J}$ and $\epsilon_{2}=-120 \cdot 10^{-20} \mathrm{~J}$. The phase diagram of the system of LC particles interacting via this potential is discussed in [24].

Bonded interactions include harmonic bond and harmonic pseudo-valent angle (introduced to mimic spacer rigidity on a coarse-grained level) contributions:

$$
V_{\text {bonded }}=\sum_{i=1}^{N_{\mathrm{b}}} k_{\mathrm{b}}\left(l_{i}-l_{0}^{k}\right)^{2}+\sum_{i=1}^{N_{\mathrm{a}}} k_{\mathrm{a}}\left(\theta_{i}-\theta_{0}\right)^{2},
$$

where $l_{i}$ and $\theta_{i}$ are instant values for $i$ th bond length and $i$ th pseudo-valent angle (defined between each three consecutive beads in a spacer), respectively, $N_{\mathrm{b}}$ and $N_{\mathrm{a}}$ being their maximum numbers. Force constants are: $k_{\mathrm{b}}=50 \cdot 10^{-20} \mathrm{~J} / \AA^{2}$ and $k_{\mathrm{a}}=20 \cdot 10^{-20} \mathrm{~J} / \mathrm{rad}^{2}$, bond length constants are: $l_{0}^{1}=14.9 \AA$ (large sphere-first sphere of spacer), $l_{0}^{2}=3.6 \AA$ (first-second sphere of spacer), $l_{0}^{3}=3.62 \AA$ (all other bonds between spheres in the spacer), $l_{0}^{4}=2.98 \AA$ (last sphere of a spacer-mesogen nearest cap center). The pseudo-valent angle constant is $\theta_{0}=\pi$.

Let me now consider possible conformations that can be observed in such model macromolecule depending on a number of attached chains $N_{\mathrm{ch}}$. Following experimental work [8, 10 17], one would expect the possibility for the rod-like, disc-like and spherulitic shapes. It is obvious that one of the crucial factors that will define the most favourable shape(s) at given $N_{\mathrm{ch}}$ is the capability of the available molecular elements of space-filling into a required form. It is also known from both experimental [8, 10] and simulation [22] works that the mesogens of adjacent molecules highly interdigitate. For the case of a rod-like conformation (in the smectic phase), one may consider the "slim rod" limit when the breadth of the molecular rod is equal to the diameter of the large sphere $\sigma_{1}$. If such two rods interdigitate, then the mesogens from both molecules cross the mid-distance cross-section of diameter $\sigma_{1}$ (shown in grey in figure 2 on the left). The condition of tight spacefilling of each molecule into a rod is reduced then to close packing of 2D discs of diameter $D$ inside the circle of diameter $\sigma_{1}$. The number of hexagonally closely packed mesogens per cross-section circle is $N^{\prime}=k \frac{\pi r_{1}^{2}}{\pi(D / 2)^{2}}=k\left(\frac{r_{1}}{D / 2}\right)^{2} \approx 30$, where $k=0.91$ is a packing fraction for 2D hexagonal lattice and $r_{1}=\sigma_{1} / 2$. Half of these (shown as blue) belong to the lower molecule only, but each molecular rod has two tails. Therefore, the number of chains per molecule in the "slim rod" limit is $N_{\text {rod }}=N^{\prime} \approx 30$. This is an estimate for the average number of chains to form a tightly space-filled rod.

Similar estimates can be performed for the case of a disc-like conformation (in the columnar phase) in a "slim disc" limit (see, figure 2 on the right). In this case, the width of the disc is equal to $\sigma_{1}=2 r_{1}$ and its radius $R_{d}$ can be estimated from the sums of bond lengths in the spacer and half a length of the mesogen, yielding $R_{d} \approx 34.3 \AA$ (the half of the mesogen length is taken into account due to mesogens interdigitation with those from six neighboring molecules). The number of closely packed mesogens on the side surface of a disc is, therefore, $N^{\prime \prime}=k \frac{2 \pi R_{d} \cdot 2 r_{1}}{\pi(D / 2)^{2}} \approx 382$. Only half of these mesogens belong to a given molecule (shown as blue discs in the cross-section region in figure 2), hence the number of chains per molecule in a "slim disc" limit is $N_{\text {disc }}=N^{\prime \prime} / 2 \approx 191$. This number, however, turns out to be unrealistic for our model, because one should take into account that the density of chains increases closer to the central sphere. Indeed, the number of closely packed grafting beads (of radius $r_{2}=\sigma_{2} / 2$ ) attached to the side surface of a small disc of a radius $r_{1}+r_{2}$ (made around a central sphere) is only $N^{*}=k \frac{2 \pi\left(r_{1}+r_{2}\right) \cdot 2 r_{1}}{\pi r_{2}^{2}} \approx 55$, four times 

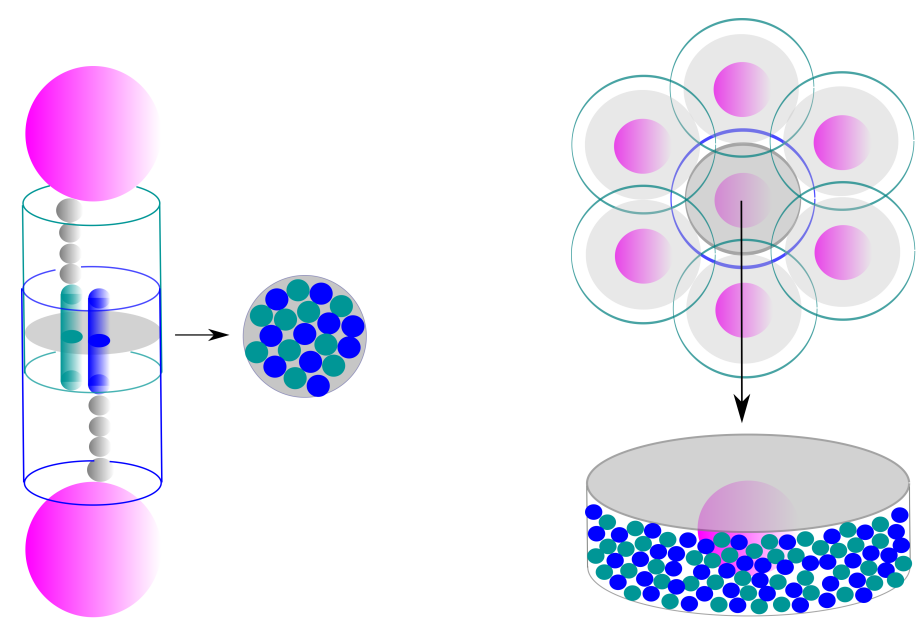

Figure 2. (Color online) On the left: cross-section region (shown as gray circle) between the tails of two adjacent molecular rods packed in an interdigitated smectic layer. The arrow points to 2D illustration of mesogens cross-sections packing inside the cross-section region (blue and green discs represent mesogens from different molecules). On the right: the same for molecular discs packed into interdigitated hexagonal columnar phase. The side surface of discs (shown below) is the cross-section region in this case, blue and green discs represent mesogens from the central and neighbouring molecules, respectively.

less than it is required for close packed mesogens on the edge surface of a disc-like molecule. Therefore, at $N_{\text {ch }}>N^{*}$ one would face a tremendous crowding of beads near the surface of a large sphere and the reasonable estimate for $N_{\text {ch }}$ to form space-filled (near the central sphere only) disc would be $N^{*} \approx 55$. For the case of spherulitic conformation, the situation is similar and the close packed external shell cannot be achieved due to limitations on the grafting density at the surface of a central sphere. The number of closely grafted polymer beads in this case is estimated as $N^{\dagger}=k \frac{4 \pi\left(r_{1}+r_{2}\right)^{2}}{\pi r_{2}^{2}} \approx 71$.

This analysis, based on space-filling of molecular elements, results in a very rough estimate for the average number of chains $N_{\mathrm{ch}} \sim 30,55,71$ that are optimal to form a rod-like, disc-like and spherulitic space-filled conformations, respectively. It leaves beyond the effect of conformational entropy, which results in swelling of both rods and discs, and this will be temperature dependent. The equilibrium conformation (and the resulting bulk morphology) will be the result of the competition between enthalpy of the mesogen-mesogen interactions and various entropic contributions to the free energy. The effects are taken into account most naturally in the CGMD simulations presented in the following section.

\section{Bulk phases, aided and spontaneous self-assembly, analysis of molec- ular conformations via CGMD simulations}

Here, I use the same coarse-grained MD approach as was used in reference [22]. This is a pretty standard MD technique only to be applied to the system with soft coarse-grained potentials, the details can be found in references [22, 26, 27]. The number of macromolecules being simulated is $N_{\text {mol }}=100$ for each case of $N_{\mathrm{ch}}=8-64$ grafted chains, the $N P T$ and $N P_{x x} P_{y y} P_{z z} T$ ensembles are used at the pressure of $53 \mathrm{~atm}$, the timestep is $20 \mathrm{fs}$ and the leap-frog integrator is employed.

It is assumed that the generic model for LC macromolecule (introduced in the previous section and shown in figure 1) is capable of self-assembling into the following bulk phases: lamellar smectic (macromolecules adopt a rod-like conformation), hexagonal columnar (macromolecules adopt a disc-like conformation) and cubic phase of possibly various symmetries. As already mentioned above, the grounds for this are to be found in both experimental [8, 10, 12, 17] and simulation [22] studies.

Slow self-assembly of LC macromolecular melts poses serious problems to computer simulations. Essential speed-up for microphase separation can be achieved by using soft potentials [e.g., equations (1)- 


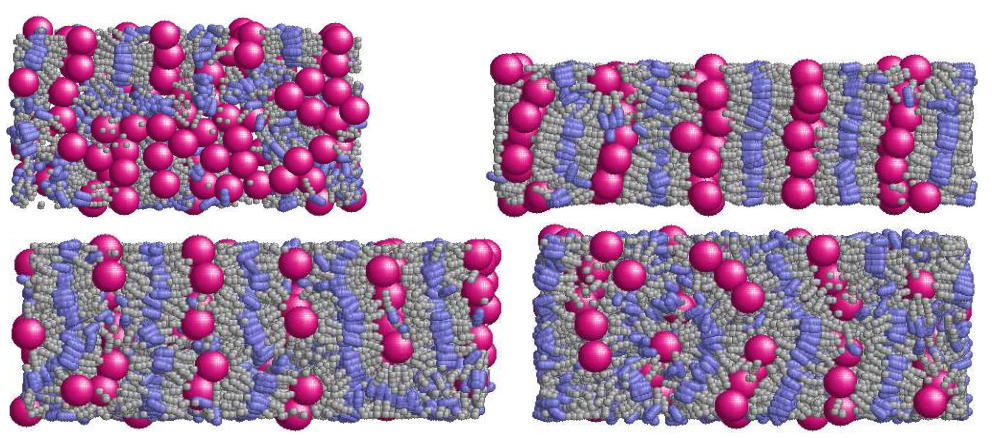

Figure 3. (Color online) Snapshots for lamellar smectic phases obtained via spontaneous self-assembly of generic model by cooling the sample from $T=500 \mathrm{~K}$ down to $450 \mathrm{~K}$ with the cooling rate of $2.5 \mathrm{~K} / \mathrm{ns}$. Top left: $N_{\mathrm{ch}}=8$, top right: $N_{\mathrm{ch}}=12$, bottom left: $N_{\mathrm{ch}}=20$, bottom right: $N_{\mathrm{ch}}=24$.

(3)], since in this case the beads are semi-transparent and may overlap and cross each other during the equilibration (see, e.g., [21, 24, 28-32]). However, for the case of the model depicted in figure1 1 the spontaneous self-assembly was still found to typically lead to the polydomain (globally isotropic) phase, both in the case of slow cooling down or slow compressing (the results for $N_{\mathrm{ch}}=32$ chains are discussed earlier [22]). Similarly to these findings, spontaneous self-assembly at a broader interval of values of $N_{\mathrm{ch}}=8-64$ turns out to be also more "hit and miss". I used relatively slow cooling, when the temperature was lowered linearly from $T=500 \mathrm{~K}$ down to $450 \mathrm{~K}$ during first $20 \mathrm{~ns}$ (cooling rate is $2.5 \mathrm{~K} / \mathrm{ns}$ ), followed by another run for $20 \mathrm{~ns}$ at fixed $T=450 \mathrm{~K}$. As the result, relatively defect-free smectic layers are found for the cases of $N_{\mathrm{ch}}=12$ and $N_{\mathrm{ch}}=20$, whereas at other values of $N_{\mathrm{ch}} \leqslant 24$, the polydomain layered structures have been obtained (see, figure 3) with the sample preparation path being the same in all cases.

There seem to be several reasons for hampering the spontaneous self-assembly of our model. The first one could be related to the annealed grafting of chains, which results in a broad uncontrolled distribution of molecular asphericity (see below) as well as may enhance microphase separation between large and small spheres, as evidenced for the case of $N_{\mathrm{ch}}=8$ (see, figure 3). The second reason is high metastability of the melt below LC transition. For instance, when the system is cooled down, once the mesogens start to form LC domains, it is locked into a random network formed by physical crosslinks between mesogens. As a result, the system is stuck in a metastable state and cannot be driven further to the global minimum morphology without applying a certain external stimulus. In real life, the perturbations of various kind do exist, e.g., random flows (when melt is poured into some vessel), centrifugal forces (when spin-coating is used), possibility to apply shear, laminar flow or external fields. These stimuli constantly "shake" the molecules in various ways and drive the melt towards the equilibrium state. Similar approaches could be also used in MD simulations.

In reference [22] the external fields acting on the mesogens were used to aid the formation of bulk phases, this approach being also adopted in our study. The external field is introduced via additional energy term:

$$
V_{i}^{\mathrm{rot}}=-F\left(\hat{e}_{i} \cdot \hat{f}\right)^{2},
$$

where $F$ is the amplitude of the field (the reduced amplitude $f$ will be defined as $F=f \cdot 10^{-20} \mathrm{~J}$ ), $\hat{e}_{i}$ is the unit vector directed along the long axis of $i$ th mesogen and $\hat{f}$ is the unit vector that defines the direction of the field. When $F>0$, the field has an uniaxial symmetry, when $F<0$, its symmetry is planar (promoting the orientation of the mesogens in a plane perpendicular to $\hat{f}$ vector). The latter case is inspired by simulations of azobenzene polymers [33, 34]. The approach can be termed as "aided self-assembly", in contrast to the spontaneous one. One should remark that the external field only promotes certain symmetry for the molecular conformations but the molecules organise themselves into a bulk phase by means of self-assembly.

The smectic-isotropic and columnar-isotropic transition temperatures are found to be in the range of $490-500 \mathrm{~K}$ and weakly dependent on the number of attached chains $N_{\mathrm{ch}}$ if $N_{\mathrm{ch}} \leqslant 40$. This is attributed 


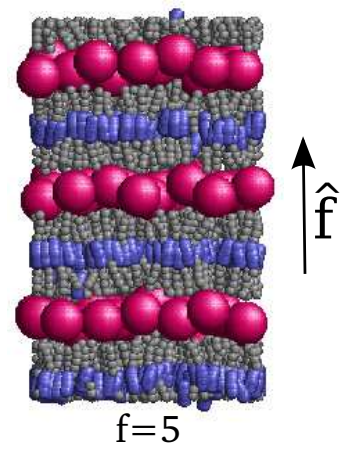

$\mathrm{N}_{\mathrm{ch}}=8$

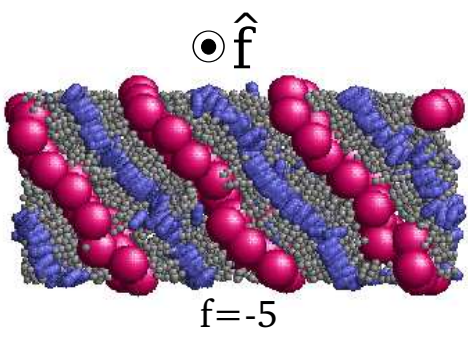

Figure 4. (Color online) Results for an aided self-assembly of generic model with $N_{\mathrm{ch}}=8$ grafted chains. Left hand frame: uniaxial aiding field, right hand frame: planar aiding field, field direction $\hat{f}$ is shown as arrow (points towards the reader in the right hand frame). Note that the same lamellar smectic phase is formed in both cases.

to the fact that the mesogen-mesogen interactions are the same in all the cases. Therefore, to search for ordered phases, the following steps are performed. First, the initial system is formed by filling the simulation box randomly by LC macromolecules with $N_{\text {ch }}$ chains directed radially out of a central sphere. Then, the short $N V T$ run is performed at $T=500 \mathrm{~K}$ with the time step of $2 \mathrm{fs}$ to remedy the beads overlapping. After that, several aided self-assembly runs of duration $20 \mathrm{~ns}$ are performed at $T=520 \mathrm{~K}$ (above the LC transition) with the timestep of $20 \mathrm{fs}$ in $N P_{x x} P_{y y} P_{z z} T$ ensemble (for more details on this ensemble, see [35]). The runs differ by the value of a reduced field strength chosen from the interval of $f=$ $[3 ; 5]$ for the uniaxial field and $f=[-5 ;-3]$ for the planar one. Finally, the following runs are performed (mostly at $T=450 \mathrm{~K}$, about $50 \mathrm{~K}$ below the LC transition) in which the external field is removed, to check on the stability of each bulk phase. All these runs are performed at non-zero external pressure, as far the system is mostly density driven (out of all the non-bonded interactions, equations (1)-(3), only the mesogen-mesogen pair potential has an attractive contribution). The pressure of 53atm is found to be quite adequate for this purpose, as was found in an earlier study [22].

At the lower end of $N_{\mathrm{ch}}$ values, the rod-like molecular conformation and bulk lamellar smectic phase are expected. The self-assembly of this phase is aided by an uniaxial field with $f>0$ at $T=520 \mathrm{~K}$. Nevertheless, for the sake of comparison, I also performed runs for $f<0$ (attempting to force a discotic conformation). In both cases, $\hat{f}$ is oriented along $Z$ axis and the runs of 10 ns duration are performed. After that, the field is removed and the system is equilibrated for another $20 \mathrm{~ns}$ at $T=450 \mathrm{~K}$. Remarkably, the same lamellar smectic morphology is obtained in both cases (of $f>0$ and $f<0$ ), the layers only differ in their arrangement with respect to the spatial axes (see, figure 4). In particular, at $f=5$ the long axes of molecular rods are directed along $Z$ axis, whereas at $f=-5$ they are confined within $X Y$ planes. In the latter case, the quasi-2D spontaneous self-assembly occurs inside these planes resulting in the formation of the smectic layers. In both simulations with uniaxial and planar fields, the rod-like conformation is observed only (the histograms will be provided below), which says in favour of the aiding field approach. Indeed, the symmetry of the field is not capable of forcing a certain conformation to occur (in this case - a discotic one), if it is not a native one for a given value of $N_{\mathrm{ch}}$. The same scenario holds for at least $N_{\text {ch }}=16$ attached chains, and in all these cases the lamellar smectic phase is observed only. At the range of values of $N_{\mathrm{ch}}=24-40$, the model displays conformational bistability, discussed earlier in [22]. In this case, the symmetry of the aiding field acts as a conformation switcher. The largest number of chains at which the smectic phase is observed is 40, higher than the close-packing estimate for the "slim rod" model (see, previous section) $N^{\prime}=30$, thus, indicating a "swollen rod" conformation. At a larger number of chains, $N_{\mathrm{ch}}=48$, the lamellar smectic phase can be forced by the field $f=5$, but it turns out to be unstable if the field is removed and the temperature reduced to $450 \mathrm{~K}$ (see, figure 5). Preliminary runs, performed for $N_{\mathrm{ch}}=48$ in a temperature range of $T=[300,500] \mathrm{K}$, indicate that the smectic-isotropic transition temperature in this case is much lower than for the case of $N_{\text {ch }}=32$, namely $T \sim 400 \mathrm{~K} v s$ $T \sim 490 \mathrm{~K}$, respectively. These effects will be covered in detail in a separate study. 

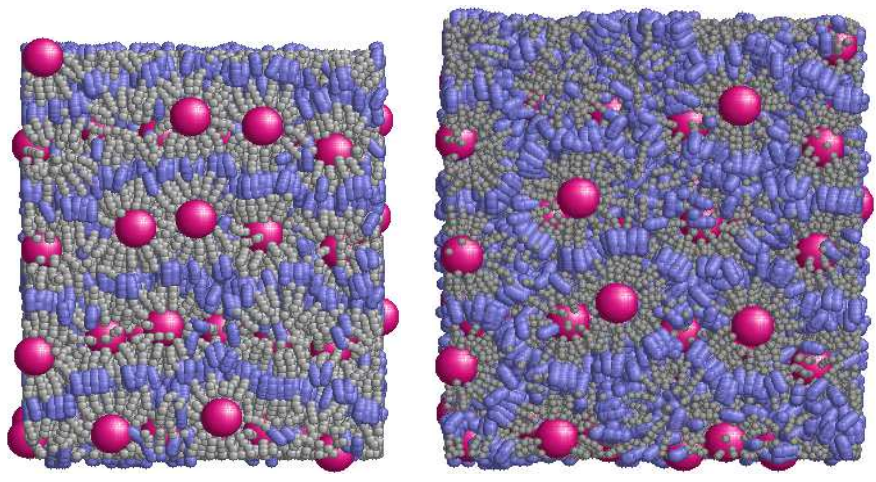

Figure 5. (Color online) Forced lamellar smectic phase for a generic model with $N_{\mathrm{ch}}=48$ chains kept by means of uniaxial field (left hand frame) and break-up of this phase when the field is switched "off" (right hand frame).

The application of the planar field with $f<0$ induces a disc-like conformation and aids self-assembly of a defect-free hexagonally packed columnar phase for $N_{\mathrm{ch}}=24-48$, including the case of $N_{\mathrm{ch}}=32$ discussed in detail in reference [22]. The properties of this phase and the snapshots are to be found there and are not repeated here. At $N_{\mathrm{ch}} \sim 56-64$, the discotic conformation transforms into a spherulitic and, as a result, the cubic phase is formed (see, figure 6). Two views of the cubic phase are shown in this figure, and on the r.h.s. one may identify the structure of swollen columns of the former columnar phase. The interval of stability for the disc-like conformation in terms of $N_{\mathrm{ch}}$ is not spanning up to the value predicted by close packing of the grafting points, $N^{*} \approx 55$, indicating not tightly packed discs.

Let me switch now to the quantitative analysis of conformations in the observed bulk phases. To do so I split the system into rods and discs and build histograms for asphericity of their conformations. First of all, the components of gyration tensor are evaluated for each $k$-th molecule:

$$
G_{\alpha \beta}^{[k]}=\frac{1}{N^{[k]}} \sum_{i=1}^{N^{[k]}}\left(r_{i, \alpha}^{[k]}-R_{\alpha}^{[k]}\right)\left(r_{i, \beta}^{[k]}-R_{\beta}^{[k]}\right), \quad \vec{R}^{[k]}=\frac{1}{N^{[k]}} \sum_{i=1}^{N^{[k]}} \vec{r}_{i}^{[k]}
$$

where $N^{[k]}$ particle centers with coordinates $r_{i, \alpha}^{[k]}$ are taken into account, $R_{\alpha}^{[k]}$ is the molecular center of mass, $\alpha, \beta$ denote Cartesian axes. To account for an extended shape of mesogens, each is replaced by a line of four centers. The eigenvalues of gyration tensor, $\lambda_{\max }^{[k]}, \lambda_{\operatorname{med}}^{[k]}$ and $\lambda_{\min }^{[k]}$ (where the indices denote
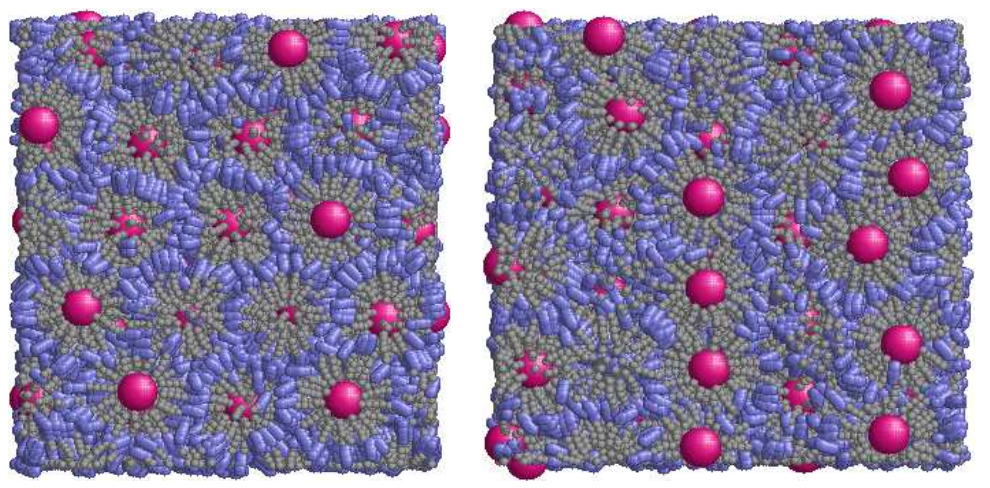

Figure 6. (Color online) Two views showing the symmetry of the cubic phase obtained as the result of either spontaneous or aided with planar field self-assembly of generic model with $N_{\mathrm{ch}}=64$ chains. The image on the right resembles columnar structure being swollen due to the change of molecular conformations from disc to a sphere. 

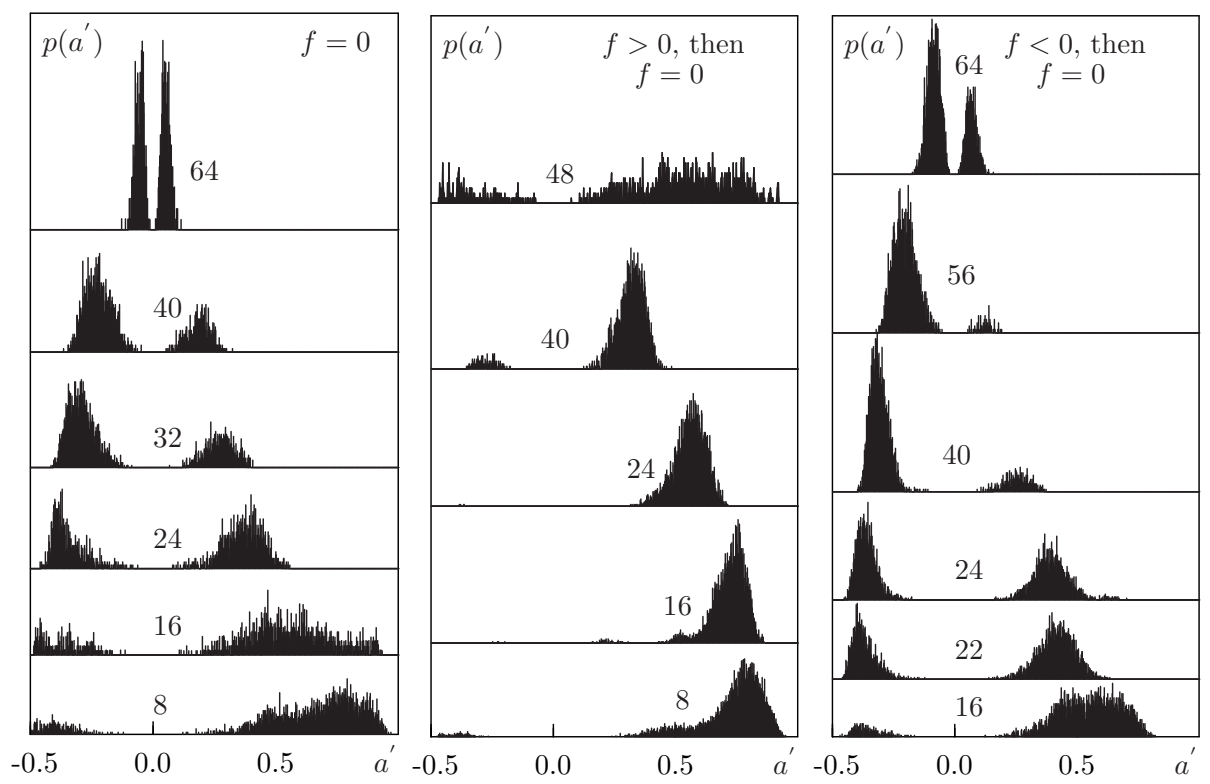

Figure 7. Histograms for the distributions of molecular asphericity $p\left(a^{\prime}\right)$ (see text for explanations) shown for a spontaneous self-assembly (left hand image), uniaxial field aided self-assembly (middle image) and planar field aided self-assembly (right hand image). Only characteristic $N_{\text {ch }}$ cases are shown in each case.

maximum, medium and minimum value, respectively) are evaluated next. These are used to introduce molecular "roddicity" (always positive):

$$
a_{\mathrm{r}}^{[k]}=\left[\lambda_{\max }^{[k]}-\frac{1}{2}\left(\lambda_{\mathrm{med}}^{[k]}+\lambda_{\min }^{[k]}\right)\right]\left[R_{g}^{[k]}\right]^{-2}
$$

and molecular “discoticity” (always negative):

$$
a_{\mathrm{d}}^{[k]}=\left[\lambda_{\min }^{[k]}-\frac{1}{2}\left(\lambda_{\text {med }}^{[k]}+\lambda_{\max }^{[k]}\right)\right]\left[R_{g}^{[k]}\right]^{-2},
$$

for each $k$ th molecule. Here, $\left[R_{g}^{[k]}\right]^{2}=\lambda_{\max }^{[k]}+\lambda_{\operatorname{med}}^{[k]}+\lambda_{\min }^{[k]}$ is squared radius of gyration. If, for a given $k$, the "roddicity" prevails, $\left|a_{\mathrm{r}}^{[k]}\right|>\left|a_{\mathrm{d}}^{[k]}\right|$, then it is classified as a rod and its asphericity is set to $a^{\prime}=a_{\mathrm{r}}^{[k]}$, otherwise the molecule is classified as a disc with its asphericity set to $a^{\prime}=a_{\mathrm{d}}^{[k]}$. As a result, the system splits into rods and discs subsystems, with their fractions $f_{\mathrm{r}}$ and $f_{\mathrm{d}}$, respectively. The histograms for $a^{\prime}$ distribution $p\left(a^{\prime}\right)$ are built over all the molecules in the system averaged over time trajectory. These are shown in figure 7 for some characteristic values of $N_{\text {ch }}$ in each case of spontaneous self-assembly (left hand frame), and self-assembly aided by an uniaxial (middle frame) and planar (right hand frame) fields. I did not include the histograms for $N_{\mathrm{ch}}=12$ and 20 (see, figure 3) into left hand frame, as the self-assembly in these two cases were rather atypical as compared with other cases of spontaneous selfassembly (these follow the route similar to an aided self-assembly).

The distributions of discoticity and roddicity are conveniently separated on these plots as far as the former is negative and the latter is positive, the values close to zero indicate spherulitic conformations. One can also see the relative weight of rod- and disc-like conformations via the height of each wing, as well and the breadth of each distribution. One may make the following observations from the histograms shown in figure 7. In the case of a polydomain phase, as a result of spontaneous self-assembly (left hand frame), rods and discs always coexist and the distributions of their asphericities are rather broad. With an increase of $N_{\mathrm{ch}}$, two maxima gradually merge into a spherulitic shape from both sides of $a^{\prime}=0$ (at about $N_{\text {ch }}=64$ and higher). The histograms for the field-aided self-assembly are essentially narrower. In the case of uniaxial field (middle frame) the discotic conformations are completely eliminated (except the 

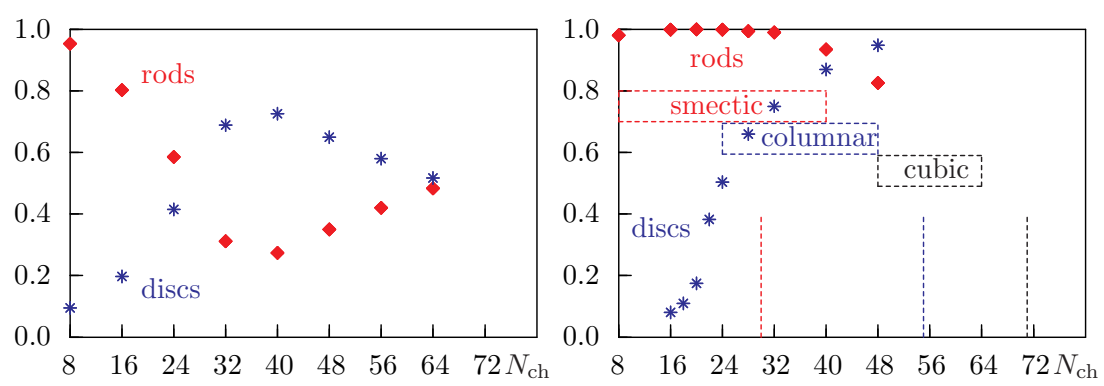

Figure 8. (Color online) Fraction of rods and discs for spontaneous self-assembly (left frame, $f_{\mathrm{r}}+f_{\mathrm{d}}=1$ ). The same properties are shown on the right but fraction of rods is shown for uniaxial field aided runs and fraction of discs - for planar field aided runs, $f_{\mathrm{r}}+f_{\mathrm{d}} \neq 1$ in this case. The figure on r.h.s. shows also the approximate phase boundaries for the smectic, columnar and cubic phases (dashed coloured horizontal text boxes) and the optimal numbers for space-filling of rod, disc and sphere from geometry estimates (vertical red, blue and black dashed lines, respectively).

case of $N_{\text {ch }}=48$ where smectic phase is not observed any more), as these are incompatible with the 1D symmetry of the aiding field. In the case of planar filed (right hand frame), which has a 2D symmetry, the rod-like conformations are not eliminated and do appear within $X Y$ plane, and are, in fact, the dominant ones at smaller values of $N_{\mathrm{ch}}$ (as discussed above for the case of $N_{\mathrm{ch}}=8$, see figure 4). With an increase of $N_{\mathrm{ch}}$ above 24, the disc-like conformations dominate. Here, I would like to remind again that the aiding field is switched "on" only at the beginning of each run, to promote the first "kick", followed by an extensive simulation with the field switched "off". The comparison of histograms for spontaneous and aided self-assembly cases reveals the effect of the aiding field in the form of conformation switching/enriching. After the required conformations are enriched, the melt is capable of self-assembling into an appropriate phase.

The fractions of rods and discs, $f_{\mathrm{r}}$ and $f_{\mathrm{d}}$, as functions of $N_{\mathrm{ch}}$ are displayed in figure 8 for various self-assembly runs. Left hand frame contains the data for a spontaneous self-assembly and it indicates a broad region for a rod-disc coexistence at intermediate values of $N_{\mathrm{ch}}$. At $N_{\mathrm{ch}}=64$, the system approaches a symmetric case with both conformations transforming into a spherulitic shape. The right hand frame contains data for $f_{\mathrm{r}}$ for uniaxial field aided self-assembly and data for $f_{\mathrm{d}}$ for planar field aided selfassembly. Therefore, $f_{\mathrm{r}}+f_{\mathrm{d}} \neq 1$ as both are obtained for different cases. One can see that the shapes of both curves are much steeper in this case as compared to the left hand frame plot indicating once more the possibility to control the molecular conformation by means of initial field of appropriate symmetry.

The comparison between the intervals with high molecular roddicity and discoticity with the intervals of stability for the smectic and columnar phase (shown as coloured text boxes in figure 8 on the right) shows their exact coincidence, thus indicating a strong correlation between the average molecular shape and the symmetry of the bulk phase. The space-filling geometrical estimates for slim rod and disc are 1.2-1.7 times larger than the approximate mid-points of the respective intervals of stability of each phase. Therefore, the real conformations considerably deviate from the "slim"-like models.

\section{Conclusions}

Computer simulations performed and discussed in this study provide some more insight on a macromolecular self-assembly of liquid crystal colloids. A generic model being used consists of a large central sphere and is modified on its surface by grafted chains each terminated by a mesogen. The focus of current study is on the role played by the surface density of chains on phase diagram and typical molecular conformations.

Simple geometry estimates based on space-filling of macromolecule into a rod-like, disc-like and spherulitic shape provided some reasonable starting point for the relation between the number of grafted chains and equilibrium conformation. Molecular dynamics simulations using soft interaction models re- 
peat the experimental evidence for the lamellar-columnar-cubic sequence of phases with an increase of surface density. I found the model being conformationally bistable at a wide range of surface density with the possibility to form either lamellar smectic or columnar phase.

Conformational analysis is performed by introducing "roddicity" and "discoticity" of their shape and, therefore, sorting the molecules at each time instance into rods and discs. The fraction of molecules in each subsystem provides some preliminary information on the distribution of their conformations. More details are provided by the histograms of their asphericity, these also shed some light on a process of macromolecular self-assembly. In this respect, the main obstacle in efficient self-assembly into a monodomain phases is seen in a lack of control over the molecular conformations. In virtually all the cases of surface density being considered, the rod- and disc-like conformations coexist and have relatively broad distribution of their asphericity.

The problem can be partially remedied by an aided self-assembly used in this study. It implies the use of an external field of certain symmetry (uniaxial, planar, etc.) which acts on the mesogens orientations to promote specific conformations (rod-, disc-like, etc.). When the bulk phase is formed, the field is removed and the system is equilibrated at a desired temperature to check for the stability of thus formed phase and to evaluate its properties. The problem of this approach is a limited choice for the symmetry of the field and a bias towards specific phase which should be known a priori. Another possible reason for, in general, poor self-assembly of this particular model could be connected with the fact that grafted chains are freely sliding on the large sphere resulting in broad distributions for molecular asphericity and, as observed in some cases, an enhanced microphase separation between large and small spheres.

This directs the following research in this area into refining the generic model towards real systems and into developing some specific techniques to drive macromolecular self-assembly.

\section{Acknowledgements}

The paper is dedicated to the 70th birthday anniversary of professor Myroslav Holovko, great scientist and teacher.

The author acknowledges participation in one of the workshops from the "Mathematics of Liquid Crystals" series by INIMS (Cambridge, UK), 8-22 March 2013 and benefited from exchange visits in the frames of EU Grant No. PIRSES 268498.

\section{References}

1. Riess G., Prog. Polym. Sci., 2003, 28, 1107; doi 10.1016/S0079-6700(03)00015-7

2. Olsen B.D., Segalman R.A., Mat. Sci. Eng. R, 2008, 62, 37; doi 10.1016/j.mser.2008.04.001

3. Zeng F., Zimmerman S.C., Chem. Rev., 1997, 97, 1681; doi 10.1021/cr9603892

4. Discher D.E., Eisenberg A., Science, 2002, 297, 967; doi 10.1126/science.1074972

5. Gittins P.J., Twyman L.J., Supramol. Chem., 2003, 15, 5; doi 10.1080/1061027031000073199

6. Percec V., Mitchell C.M., Cho W.-D., Uchida S., Glodde M., Ungar G., Zeng X., Liu Y., Balagurusamy V.S.K., Heiney P.A., J. Am. Chem. Soc., 2004, 126, 6078; doi 10.1021/ja049846j

7. Tschierske C., J. Mater. Chem., 2001, 11, 2647; doi 10.1039/b102914m

8. Saez I.M., Goodby J.W., J. Mater. Chem., 2005, 15, 26; doi 10.1039/b413416h

9. Tschierske C., Chem. Soc. Rev., 2007, 36, 1930; doi 10.1039/b615517k

10. Saez I.M., Goodby J.W., Struct. Bond., Liquid Crystalline Functional Assemblies and Their Supramolecular Structures, 2008, 128, 1; doi 10.1007/430_2007_077

11. Draper M., Saez I.M., Cowling S.J., Gai P., Heinrich B., Donnio B., Guillon D., Goodby J.W., Adv. Funct. Mater., 2011, 21, 1260; doi 10.1002/adfm.201001606

12. Bisoyi H.K., Kumar S., Chem. Soc. Rev., 2011, 40, 306; doi 10.1039/b901793n

13. Ponomarenko S.A., Boiko N.I., Shibaev V.P., Richardson R., Whitehouse I., Rebrov E., Muzafarov A., Macromolecules, 2000, 33, 5549; doi 10.1021/ma0001032

14. Agina E.V., Boiko N.I., Richardson R.M., Ostrovskii B.I., Shibaev V.P., Rebrov E.A., Muzafarov A.M., Polym. Sci. Ser. A, 2007, 49, 412; doi 10.1134/S0965545X07040086

15. Wojcik M., Lewandowski W., Matraszek J., Mieczkowski J., Borysiuk J., Pociecha D., Gorecka E., Angew. Chem. Int. Edit., 2009, 48, 5167; doi 10.1002/anie.200901206. 
16. Wojcik M., Kolpaczynska M., Pociecha D., Mieczkowski J., Gorecka E., Soft Matter, 2010, 6, 5397; doi $10.1039 / \mathrm{c} 0 \mathrm{sm} 00539 \mathrm{~h}$

17. Wojcik M., Gora M., Mieczkowski J., Romiszewski J., Gorecka E., Soft Matter, 2011, 7, 10561; doi $10.1039 / \mathrm{c} 1 \mathrm{sm} 06436 \mathrm{c}$

18. Wilson M.R., Ilnytskyi J.M., Stimson L.M., J. Chem. Phys., 2003, 119, 3509; doi 10.1063/1.1588292

19. Mazo M.A., Shamaev M.Yu., Balabaev N.K., Darinskii A.A., Neelov I.M., Phys. Chem. Chem. Phys., 2004, 6, 1285; doi $10.1039 / \mathrm{b} 311131 \mathrm{~h}$

20. Markelov D.A., Mazo M.A., Balabaev N.K., Gotlib Yu.Ya., Polym. Sci. Ser. A, 2013, 55, 53; doi $10.1134 /$ S0965545X13010045

21. Hughes Z.E., Wilson M.R., Stimson L.M., Soft Matter, 2005, 1, 436; doi 10.1039/b511082c

22. Ilnytskyi J.M., Lintuvuori J., Wilson M.R., Condens. Matter Phys., 2010, 13, 33001; doi 10.5488/CMP.13.33001.

23. Orlandi S., Zannoni C., Mol. Cryst. Liq. Cryst., 2013, 573, 1; doi 10.1080/15421406.2012.763213

24. Lintuvuori J.S., Wilson M.R., J. Chem. Phys., 2008, 128, 044906; doi 10.1063/1.2825292

25. Earl D.J., Ilnytskyi J., Wilson M.R., Mol. Phys., 2001, 99, 1719; doi 10.1080/00268970110069551

26. Ilnytskyi J., Wilson M.R., Comput. Phys. Commun., 2001, 134, 23; doi 10.1016/S0010-4655(00)00187-9

27. Ilnytskyi J., Wilson M.R., Comput. Phys. Commun., 2002, 148, 43; doi 10.1016/S0010-4655(02)00467-8

28. Groot R.D., Madden T.J., J. Chem. Phys., 1998, 108, 8713; doi $10.1063 / 1.476300$

29. Ilnytskyi J., Patsahan T., Holovko M., Krouskop P., Makowski M., Macromolecules, 2008, 41, 9904; doi $10.1021 / \mathrm{ma} 801045 \mathrm{z}$

30. Bates M., Walker M., Soft Matter, 2009, 5, 346; doi 10.1039/b813015a

31. Bates M.A., Walker M., Phys. Chem. Chem. Phys., 2009, 11, 1893; doi $10.1039 / \mathrm{b} 818926 \mathrm{a}$

32. Lintuvuori J.S., Wilson M.R., Phys. Chem. Chem. Phys., 2009, 11, 2116; doi 10.1039/b818616b

33. Ilnytskyi J.M., Neher D., Saphiannikova M., Condens. Matter Phys., 2006, 9, 87; doi 10.5488/CMP.9.1.87

34. Ilnytskyi J.M., Neher D., Saphiannikova M., J. Chem. Phys., 2011, 135, 044901; doi 10.1063/1.3614499

35. Ilnytskyi J.M., Neher D., J. Chem. Phys., 2007, 126, 174905; doi 10.1063/1.2712438

\title{
Взаємозв'язок між поверхневою густиною рідкокристалічної макромолекули та симетрією їі саморганізованої фази: дослідження за допомогою методу огрубленої молекулярної динаміки
}

\author{
Я.М. Ільницький \\ Інститут фізики конденсованих систем НАН України, вул. Свєнціцького, 1, 79011 Львів, Україна
}

Розглянуто узагальнену модель, придатну для опису об'ємного впорядкування рідкокристалічних (РК) макромолекул (наприклад, РК дендримерів; наночастинок золота, модифікованих полімерними ланцюжками із кінцевими РК групами тощо). Дослідження концентрується на взаємозв'язку між кількістю приєднаних ланцюжків $N_{\text {ch }}$ та симетрією впорядкованої фази. Використовуючи прості геометричні обчислення спочатку оцінено інтервали стабільності для стержне-, диско- та сферо-подібних молекулярних конформацій залежно від $N_{\mathrm{ch}}$. Далі виконано моделювання за допомогою молекулярної динаміки для спонтанного та керованого самовпорядкування РК макромолекул в об'ємні фази. Під час спонтанного самовпорядкування шляхом аналізу гістограм для молекулярної асферичності виявлено співіснування стержнета диско-подібних конформацій в широкому інтервалі $N_{\mathrm{ch}}$, що перешкоджає формуванню бездефектних структур. Використання одновісного або планарного керуючих полів суттєво покращує самовпорядкування відповідних монодоменних фаз шляхом селекції конформацій з відповідною симетрією. Сильна залежність між формою молекули та симетрією фази, яка спостерігається експериментально, також виявляється і при моделюванні - через співпадіння інтервалів стабільності відповідних конформацій та об'ємних фаз.

Ключові слова: макромолекули, рідкі кристали, самовпорядкування, молекулярна динаміка 